
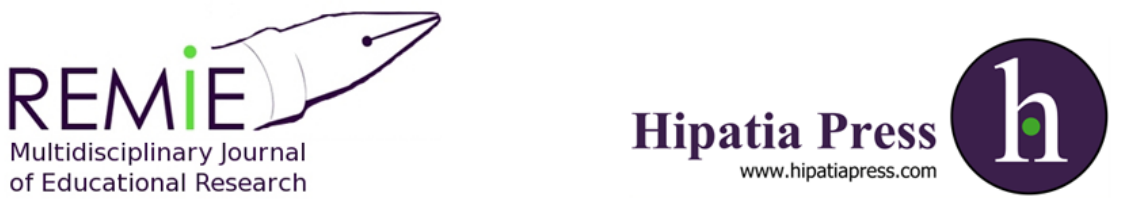

Instructions for authors, subscriptions and further details:

http://remie.hipatiapress.com

\title{
Aprendizaje-Servicio como Estrategia Metodológica para Impulsar Procesos de Educación Expandida.
}

Domingo Mayor 1

1) Universidad de Almería

Date of publication: February 15th, 2020

Edition period: October 2019 - February 2020

To cite this article: Mayor, D. (2020). Aprendizaje-Servicio como estrategia metodológica para impulsar procesos de educación expandida.

Multidisciplinary Journal of Educational Research, 10(1), 47-74. doi:

10.4471/remie.2020.4562

To link this article: http://dx.doi.org/10.447/remie.2020.4562

PLEASE SCROLL DOWN FOR ARTICLE

The terms and conditions of use are related to the Open Journal System and to Creative Commons Attribution License (CC-BY). 


\section{Aprendizaje-Servicio como Estrategia Metodológica para Impulsar Procesos de Educación Expandida}

Domingo Mayor

Universidad de Almería

(Recibido: 8 Agosto 2019; Aceptado: 26 Enero 2020; Publicado: 15 Febrero 2020)

\section{Resumen}

En este estudio se exponen algunos de los resultados de una investigación centrada en la percepción del estudiantado universitario, del grado de Educación Social, y el coordinador de un proyecto de Aprendizaje-Servicio que se realizó en un barrio socialmente desfavorecido. En el mismo participaron: estudiantado y profesor universitario, alumnado de secundaria y primaria y profesionales de distintos ámbitos socioeducativos. Para ello se planteó una investigación de enfoque mixto, utilizando el estudio de caso de carácter exploratorio como método de investigación. Como técnicas de recogida de datos se usaron la observación participante, el análisis de documentos, las entrevistas semiestructuradas individuales y grupales y un cuestionario ad hoc, diseñado por el profesor. Los resultados obtenidos permiten constatar los efectos producidos en distintas dimensiones relacionadas los proyectos de Aprendizaje-Servicio: la vinculación teoría-práctica, los aprendizajes alcanzados y el desarrollo del compromiso social del alumnado universitario. Estos hallazgos evidencian la importancia de esta metodología para la creación de escenarios pedagógicos expandidos que favorecen la retroalimentación entre teoría-práctica en contextos reales, la importancia de la reflexión en la adquisición de los aprendizajes experienciales y el análisis de la realidad y la creación de acciones socioeducativas como ejes para el desarrollo del compromiso social.

Keywords: aprendizaje-servicio, educación expandida, conexión teoría-práctica, aprendizaje experiencial, compromiso social 


\section{Service Learning as a Methodological Strategy for Promoting Expanded Education}

Domingo Mayor

University of Almería

(Received: 8th August 2019; Accepted: 26th January 2020; Published: 15th February 2020)

\section{Abstract}

This paper describes some of the results of a study on the perception of Social Education degree students and the coordinator of a Service-Learning project carried out in a socially disadvantaged neighborhood. Participants in it were the student body and a university professor, primary and secondary school students, and professionals from various socioeducational fields. To do this, a mixed approach using the exploratory case study was posed as the research method. Data acquisition techniques were participant observation, document analysis, individual and group semi-structured interviews and an ad hoc questionnaire designed by the professor. The results confirmed the effects on various dimensions related to Service-Learning Projects: The theory-practice link, learning achieved and development of social commitment of the university students. These findings demonstrate the importance of this methodology for creating expanded pedagogical scenarios favoring theory-practice feedback in real contexts, the importance of reflecting on acquisition of experiential learning and analysis of the reality and creation of socio-educational action as axes for developing social commitment.

Palabras clave: service-learning, expanded education, theory-practice connection, experiential learning, social commitment. 


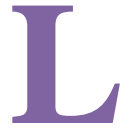

a institución escolar, como construcción sociohistórica, se crea para dar respuesta a las necesidades sociales, políticas, económicas y culturales del siglo XIX, cumpliendo dos grandes funciones: la preparación de las nuevas generaciones para su incorporación futura al mundo del trabajo y la formación de la ciudadanía para su intervención en la vida pública (Gimeno, 2005; Pérez, 2012). Para su configuración organizativa, funcional y arquitectónica se siguió el modelo fábrica, donde los valores dominantes eran la homogeneidad, uniformidad, la disciplina, el control, el conocimiento memorístico distanciado de los problemas reales que vivía el alumnado, etc., alejándose así de las circunstancias cambiantes que rodeaban el mundo vital de los menores, en particular, y de la sociedad, en general.

Este distanciamiento, ensimismamiento o endogamia, obstaculizaba su adaptación a las transformaciones que se estaban produciendo en el ámbito científico y tecnológico, en la actividad laboral, en la estructura familiar y en los valores, costumbres relacionadas con la vida cotidiana, así como la diversificación y ampliación de la demanda cultural, el desarrollo de la pedagogía y de las ciencias de la educación (Carbonell, 2016; Puig, J. M., Batlle, R., Bosch, C. \& Palos, 2007). Dichas dificultades, para responder a las nuevas realidades, fueron criticadas desde diferentes posiciones por autores que defendían la desescolarización de la sociedad (Trilla, 2004; Trilla), así como por organismos internacionales (UNESCO. , 1996) que, a través de diversos informes encargados a expertos, fueron radiografiando la realidad educativa mundial a partir de la década de los 60 .

El reconocimiento progresivo de los espacios múltiples y heterogéneos, pertenecientes a la educación formal, no formal e informal, que iban configurando el nuevo universo educativo, por parte de instituciones internacionales y nacionales y estudiosos de la educación; su formalización disciplinar en áreas de conocimiento como la pedagogía, sociología, psicología, etc.; el potencial educativo de las tecnologías de la información y la comunicación; la necesidad de rentabilizar los recursos públicos y privados insertos en un territorio; la incapacidad de la escuela para hacer frente, por sí sola, a todas las demandas sociales vinculadas a la instrucción y formación integral, etc., son algunos de los elementos que han incidido, por un lado, en la reconfiguración del concepto de educación, ampliando sus 


\section{Mayor - Aprendizaje Servicio y Educación Expandida}

contornos más allá del entorno escolar y, por otro, en la búsqueda de nuevas formas de hacer y relacionarse, orientadas a promover sinergias para aprovechar el potencial educativo que encierran las comunidades físicas y virtuales donde la escuela es considerada como un nodo más del conjunto de nodos que conforman el tejido de las redes por donde transitan las personas y grupos humanos a lo largo y ancho de su vida (Carbonell, 2016; Martínez, J. B. \& Fernández, 2018; Mayor, 2018; Subirats, 2002).

La conexión entre la escuela y la comunidad es una idea que ha ido madurando debido a los procesos sociales y educativos que se han ido entrelazando, así como a la identificación y análisis de diferentes prácticas educativas que se estaban implementando fuera del entorno escolar y a la evolución del pensamiento pedagógico (Carbonell, 2016; Caride, 2006; Dewey, 1950; Freire, 1994; Olsen, 1951) que pasó de observar y analizar los fenómenos educativos en el ámbito escolar, a significar dichos fenómenos desde una perspectiva holística e integradora. En este sentido, Martínez y Fernández (Martínez, J. B. \& Fernández, p. 13) exponen:

Los contextos donde se producen hoy los aprendizajes han desbordado las fronteras institucionales de la educación forma, no formal e informal, de manera que se aprende en cualquier tiempo y lugar a través de agentes, dispositivos y procedimientos de innovación permanente. La educación expandida se filtra por los huecos de las instituciones, se expande por las grietas de lo formal y burocrático cuando se introducen o sacan del aula experiencias que se saltan las secuencias convencionales de objetivos operativos, horarios inflexibles, espacios de aislamiento o conocimientos disciplinares fragmentados.

Acciones pedagógicas expandidas que aluden a la exploración de los límites más allá de lo que hoy conocemos como educación formal, ampliando los contornos y situándolos un poco más lejos, para buscar espacios de intersección con otras prácticas y disciplinas (ZEMOS., 2012). Todo ello va generando nuevas ecologías de aprendizajes (Coll, 2013), surgidas como consecuencia de los cambios sociales, políticos, económicos, tecnológicos y culturales que atraviesan las sociedades actuales, planteando nuevos retos y desafíos inéditos a la educación escolar, ya que se vincula a un modelo educativo (Ver tabla 1) emergente en el que la acción educativa está distribuida entre diferentes escenarios y agentes. 
Tabla 1.

Ecología de aprendizaje. Nuevo modelo educativo

\begin{tabular}{|c|c|c|}
\hline Dimensiones & Escolarización universal & $\begin{array}{l}\text { Acción educativa distribuida e } \\
\text { interconectada. }\end{array}$ \\
\hline Dónde y quién & $\begin{array}{l}\text {-Instituciones educativas. } \\
\text {-Profesionales de la } \\
\text { educación. }\end{array}$ & $\begin{array}{l}\text {-Multiplicidad de escenarios, } \\
\text { físicos y virtuales, y agentes } \\
\text { educativos. }\end{array}$ \\
\hline Cuándo & -Al inicio de la vida & $\begin{array}{l}\text {-Necesidades de aprendizaje a lo } \\
\text { largo de la vida. }\end{array}$ \\
\hline Qué & $\begin{array}{l}\text {-Saberes culturales } \\
\text { estables socialmente } \\
\text { valorados. } \\
\end{array}$ & $\begin{array}{l}\text {-Competencias y saberes inestables, } \\
\text { inciertos y cambiantes. }\end{array}$ \\
\hline Para qué & $\begin{array}{l}\text {-Para poder desarrollar } \\
\text { posteriormente un } \\
\text { proyecto de vida personal, } \\
\text { profesional y social. }\end{array}$ & $\begin{array}{l}\text {-Para formar a personas } \\
\text { competentes, capaces de seguir } \\
\text { aprendiendo en distintos momentos } \\
\text { de la vida. }\end{array}$ \\
\hline Cómo & $\begin{array}{l}\text {-Mediante la acción } \\
\text { educativa intencional, } \\
\text { sistemática y planificada } \\
\text { (enseñanza). } \\
\text {-Predominio de las } \\
\text { tecnologías basadas en la } \\
\text { lengua escrita y las } \\
\text { competencias exigidas } \\
\text { para su uso (leer y escribir } \\
\text { para aprender). }\end{array}$ & $\begin{array}{l}\text {-Participación en comunidades de } \\
\text { interés, práctica y aprendizaje. } \\
\text {-Utilización de distintos lenguajes y } \\
\text { formatos de representación de la } \\
\text { información. } \\
\text {-Saberes procedentes de distintos } \\
\text { ámbitos del conocimiento y de la } \\
\text { cultura popular. } \\
\text {-Las TIC como una vía de acceso a } \\
\text { la información y conocimiento. }\end{array}$ \\
\hline
\end{tabular}

Fuente: elaboración propia a partir del trabajo de Coll (Coll).

En el escenario de la ciudad se vienen implementando programas que responden al modelo anterior. Las denominadas Ciudades Educadoras (Asociación Internacional de Ciudades Educadoras , 2014) son acciones educativas de carácter transversal y multidisciplinar que se estructuran con la finalidad aprovechar el capital social y cultural de los diversos agentes y agencias (actores, equipamientos, espacios de ocio, servicios públicos, entidades sociales, sindicatos, etc.), diseminados en el territorio, con influencia significativa en la formación de las nuevas generaciones. 


\section{Mayor-Aprendizaje Servicio y Educación Expandida}

En el ámbito de la educación formal, desde posiciones progresistas que apuestan por la justicia social, se plantea la necesidad de crear espacios de intersección/espacios híbridos donde los discursos, prácticas y experiencias procedentes del contexto académico convergen con saberes y prácticas derivadas de otras áreas de conocimiento y de la cultura popular. Ello representa un cambio de paradigma en la epistemología de los programas de formación del profesorado (Freire, 2002; García, T., Sánchez, M. S. \& Mayor, 2012; Zeichner, 2010), ya que implica pasar de la transmisión unidireccional en el aula de información descontextualizada y con valor de cambio a la construcción colectiva de conocimientos en contextos reales con valor de uso.

En línea con lo anterior se vienen realizando acciones educativas expandidas que posibilitan la creación de espacios de intersección entre agencias y agentes pertenecientes al dispositivo escolar y a instituciones con potencial educativo insertadas en la comunidad: proyectos educativos de entorno, planes de desarrollo comunitario, comunidades de aprendizaje, Aprendizaje-Servicio, etc. (Mayor, D. \& Rodríguez, 2016; Muñoz, J. L., Rodríguez, D. \& Barrera, 2013).

De las prácticas mencionadas anteriormente nos vamos a detener en el Aprendizaje-Servicio (ApS), ya que ha sido la metodología implementando en el proyecto analizado.

\section{Aprendizaje-Servicio como Espacio de Intersección entre Agentes Procedentes de Diversos Ámbitos del Saber}

Aunque la expresión Service-learning (denominación acuñada en el ámbito anglosajón) fue usada por primera vez en el año 1967 por William Ramsay, Robert Sigmon y Michael Hart para describir un proyecto de desarrollo local llevado a cabo por estudiantes y profesorado universitario junto a organizaciones sociales de Tennessee-EE. UU- (Máximo, 2010), sus antecedentes históricos se sitúan en épocas anteriores y en contextos culturales distintos.

En la actualidad, las prácticas de ApS, se vienen desarrollando en contextos culturales muy diversos (EE. UU, México, Argentina, Holanda, Reino Unido, Alemania, Escocia, Suráfrica... y España), designándolo con diferentes nomenclaturas (pasantías cívicas, aprendizaje activo en la 
comunidad, servicio social curricular, aprendizaje-servicio solidario, etc.) y con desigual nivel de institucionalización en los distintos niveles educativos.

El vocablo ApS es utilizado para significarlo como metodología educativa experiencial, como práctica formativa o filosofía práctica (Puig, J. M. \& Palos, 2006). Como objeto de estudio puede ser analizado desde las múltiples dimensiones que configuran cada uno de los proyectos implementados en contextos singulares: marco teórico que los sustentan, aprendizajes previstos, objetivos del servicio, relaciones entre agencias y agentes, ámbitos de aplicación, participantes, niveles de implicación, resultados, etc. Todo ello sirve para dar cuenta de este objeto de estudio de su naturaleza multidimensional e interdisciplinar (Mayor, 2018).

Las investigaciones realizadas en las últimas décadas han posibilitado un acuerdo internacional sobre los ejes que estructuran al ApS: a) la conexión entre los aprendizajes académicos (educación formal) o contenidos formativos (educación no formal) con el servicio a la comunidad; b) Un nivel fuerte de participación de las personas implicadas en las distintas fases del proyecto: diseño, implementación y evaluación; y c) El proyecto puesto en acción tiene que ir dirigido a dar respuesta a una necesidad sentida por la comunidad (Mayor, D. \& Rodríguez, 2017; Montes, R., Tapia, M., \& Yaber, 2011).

De las múltiples definiciones existentes en la literatura relacionada con este tópico vamos a utilizar la planteada por Puig y Palos, ya que, por un lado, recoge los 3 ejes estructurales que vertebran esta práctica experiencial y, por otro lado, expresa el potencial educativo de esta metodología para generar espacios formativos de educación expandida: "es una propuesta educativa que combina procesos de aprendizaje y de servicio a la comunidad en un solo proyecto bien articulado en el que los participantes se forman al trabajar sobre necesidades reales del entorno con el objetivo de mejorarlo" (Puig, J. M. \& Palos, p. 61).

El aumento constante de este tipo de experiencias a nivel mundial ha llevado aparejado también un incremento del interés por indagar en sus potencialidades pedagógicas y en el impacto producido en los y las participantes, las entidades sociales y el nivel de institucionalización (Furco, 2007).

Los estudios realizados para analizar la influencia de las prácticas de ApS en los agentes implicados, utilizan diferentes enfoques metodológicos 


\section{Mayor-Aprendizaje Servicio y Educación Expandida}

(cuantitativo y/o cualitativo) e instrumentos de recogida de información diversos: cuestionarios, entrevistas, observación, etc. (Chiva-Bartoll, O. \& Gíl-Gómez, 2018). La mayoría de las investigaciones están dirigidas por profesorado universitario con el propósito de escrutar la incidencia de los proyectos en los y las discentes. En esta línea, Furco (Furco) expone que el $85 \%$ de los estudios realizados hasta hoy se refieren a cómo el ApS tiene impacto sobre distintos aspectos relacionados con el alumnado, docentes, instituciones educativas y comunidad, pero, sobre todo, se han focalizado -el $80 \%$ - en el impacto manifestado en diversas dimensiones conectadas con el estudiantado. Los resultados de las investigaciones también evidencian que el ApS tiene efectos positivos en el estudiantado en cinco áreas: actitud hacia uno mismo, actitudes hacia la universidad y el aprendizaje, compromiso cívico, competencias sociales y rendimiento académico (Furco, 2003; RuizCorbella, M. \& García-Gutiérrez, 2019; Mayor, 2018; Mella, I. Santos, M. A. \& Malherio, 2015; Rodríguez, M. \& Ordoñez, 2015). Asimismo, influye positivamente en satisfacción del alumnado relacionada con la percepción que tienen sobre la adquisición de aprendizajes auténticos, sentirse protagonistas y posibilidad de vincular teoría y la práctica (Folgueiras, P., Luna, E. \& Escofet,, 2013; Mayor, 2018).

En cuanto al impacto del ApS en el profesorado, Jouannet, Ponce y Contreras (Jouannet, C., Ponce, C. \& Contreras) indagan, por un lado, en las características de los profesores que se integran y permanecen realizando sus cursos con la metodología de ApS en la Universidad Católica (Chile) y, por otro, en su percepción respecto del impacto que la implementación de esta metodología ha tenido sobre su rol docente. Los resultados alcanzados evidencian impactos positivos en cinco dimensiones de su quehacer docente: relación con la sociedad; conocimientos disciplinares; docencia; valores, actitudes y habilidades y percepción sobre sí mismos.

Los estudios realizados para evaluar el impacto en los socios comunitario Contreras et al. (Contreras) ponen de manifiesto que los socios comunitarios (ayuntamientos, fundaciones sin ánimo de lucro y centros educativos de primaria y secundaria) se muestran satisfechos con los servicios recibidos, valorando positivamente la relación con la universidad, ya que les provee algunos servicios realizados por el estudiantado, les posibilita una red de contactos con expertos en distintas temáticas y le ofrece la posibilidad de obtener una mirada externa y crítica de su trabajo. Además, resaltan que el 
servicio recibido responde a una necesidad sentida por la organización y que el producto dejado por los estudiantes es útil para la organización.

Teniendo en cuenta las cuestiones señaladas anteriormente, en este trabajo se presentan los resultados del estudio de un proyecto de ApS en el contexto comunitario que tenía como propósito impulsar la participación del alumnado de diversos niveles educativos y profesionales de distintos ámbitos profesionales en actividades formativas que requerían la contextualización, por parte del estudiantado universitario, de los contenidos teóricos de una asignatura a situaciones problemáticas de la vida real. $\mathrm{Su}$ objetivo estuvo centrado en el análisis de la percepción del alumnado universitario y del coordinador de las acciones formativas sobre disímiles dimensiones relacionas con su implicación en el proyecto de ApS.

\section{Metodología}

\section{Contexto de la Investigación}

El proyecto estudiado se desarrolló en un barrio de Almería (España), catalogado por la Junta de Andalucía como socialmente desfavorecido debido a las siguientes circunstancias: a) Más del $70 \%$ de la población en edad de trabajar se encuentra en situación de desempleo; b) El nivel de absentismo y abandono escolar supera el 30\%; c) De las 8200 personas que habitan en el barrio, el $70 \%$ son migrantes, sobre todo proceden del Marruecos; d) El alto nivel de conflictividad entre las distintas etnias; e) El deterioro del parque público de viviendas y de los espacios públicos, etc. En el mismo intervienen profesionales pertenecientes instituciones públicas y entidades sociales con la finalidad de mejorar la vida de la población. También se involucraron profesionales que desempeñan sus funciones en otros contextos de la ciudad. En cuanto al estudiantado universitario, pertenecía al programa de estudios del grado en Educación Social, concretamente cursaba la asignatura Dirección y Gestión Pedagógica de Organizaciones Socioeducativas, una de las materias obligatorias del tercer curso, que tiene como finalidad: "desarrollar procedimientos en la formación de los estudiantes que les permitan comprender, analizar y cuestionar las organizaciones y su protagonismo en las instituciones socioeducativas, entendiendo la organización como construcción de sus participantes dentro de un contexto sociocultural determinado" (Universidad de Almería., 2018). 
El proyecto socioeducativo analizado, articulado por la metodología de ApS, se estructuraba en dos fases:

1. Formación en contenidos conceptuales, procedimentales y actitudinales impartidos por profesionales procedentes de diversos ámbitos profesionales: salud comunitaria, pedagogía del hábitat, universidad, Aprendizaje-Servicio, fotografía, música, poesía, biodanza, etc., así como el conocimiento de proyectos socioeducativos puestos en acción por las entidades sociales e instituciones públicas que trabajan en el territorio;

2. Diseño, implementación y evaluación de proyectos de ApS por parte del alumnado universitario y de educación secundaria, puestos en acción con el alumnado de un centro público de educación primaria y en la Empresa Pública de Vivienda de la Junta de Andalucía (Análisis documentos).

La acción socioeducativa de ApS se desarrolló durante 3 meses fuera del entorno universitario y, en el mismo, participaron voluntariamente 25 de los 61 alumnos matriculados. Las tareas académicas que asumieron fueron: a) Cada grupo de 4 estudiantes, contando con la supervisión del profesor y del coordinador del proyecto, tenía que diseñar un proyecto de ApS teniendo en cuenta los contenidos teóricos de la materia y la necesidad social a la que querían dar respuesta; y b) Elaboración de un diario individual reflexivo con el objetivo de ir narrando los aspectos significativos que iban emergiendo, así como las potencialidades, debilidades y propuestas de mejora de los distintos aspectos que configuraban el proyecto. Ambos instrumentos, proyecto y diario reflexivo, se contemplaron como parte de la evaluación continua y como pruebas a calificar con influencia en la nota final.

\section{Enfoque Metodológico}

En función del propósito de la investigación, se llevó a cabo una metodología de naturaleza cualitativa con el interés de tratar de identificar la complejidad del fenómeno estudiado, su estructura dinámica, aquella que da razón plena de su comportamiento y manifestaciones, ya que interesaba 
conocer lo idiosincrásico, lo particular y lo único del mismo desde la perspectiva de los participantes (Guba, 1989).

Para profundizar en el fenómeno educativo objeto de investigación, se utilizó el estudio de caso mixto de carácter exploratorio, con el propósito de comprender y explicar la idiosincrasia y complejidad de un fenómeno singular (Simons, 2011): la realización de una de acción educativa en el ámbito comunitario orientada a mejorar aspectos de la realidad, en la cual participaron agencias y agentes procedentes del ámbito de la educación formal y no formal. Acción que favoreció el intercambio de saberes y la conexión, por parte del estudiando universitario, de los contenidos teóricos de la asignatura con las actividades derivadas del servicio a la comunidad.

\section{Participantes}

Durante la realización del proyecto de implicaron, por un lado, profesionales procedentes de diferentes instituciones y ámbitos profesionales: salud, vivienda, entidades sociales, universidad, fotografía, poesía, música, biodanza, maestra de formación de adultos, directora de un centro de Educación Infantil y dos profesores/as de Educación Primaria y, por otro, alumnado de la universidad, de Educación Secundaria y de Educación Primaria.

Tabla 2.

Relación de personas implicadas en el proyecto

\begin{tabular}{ll}
\hline Número & Procedencia \\
\hline 25 & Alumnas/os del grado en Educación Social \\
\hline 1 & Profesora universitaria \\
\hline 18 & Alumnos/as de Educación Secundaria Obligatoria \\
\hline 12 & Profesores/as de Educación Primaria: \\
\hline 1 & Alumnos/as de Educación Primaria \\
\hline 16 & Coordinador del programa formativo: Educador Social \\
& $\begin{array}{l}\text { Dinamizadoras/es procedentes de distintos ámbitos:1: vivienda, 2: } \\
\text { salud, 1: universidad,5: maestras de infantil, primaria y adultos, 1: } \\
\text { biodanza, 1: música, 1: poesía, 1: fotografía, 1: TIC, 2: Asociaciones } \\
\text { del barrio }\end{array}$ \\
\hline
\end{tabular}

Fuente: elaboración propia. 


\section{Instrumentos y Procedimiento}

El proceso de estudio se realizó a lo largo de tres meses, para ello se utilizaron diversas técnicas cualitativas y cuantitativas (Wood, P. \& Smith, 2018) con diferentes propósitos, en distintos momentos y agentes implicados:

- Observación participante: en los distintos escenarios donde tuvo lugar la formación inicial y la implementación de los proyectos llevados a cabo por el alumnado de la universidad y de secundaria. Recogidas en notas de campo, han constituido el Diario de Observación del investigador.

- Cuestionario ad hoc de tipo mixto dirigido al alumnado universitario con el propósito de conocer su percepción sobre distintas dimensiones del proyecto: aprendizajes alcanzados, nivel de satisfacción obtenido, tipo de relaciones mantenidas con los profesionales, dificultades y potencialidades experimentadas, etc. El mismo estaba configurado por 25 cuestiones en las que se combinaban preguntas de escala Likert de corte cuantitativo con preguntas abiertas de carácter cualitativo.

- Entrevista semiestructurada grupal: dirigidas al alumnado de la universidad, con el objetivo de contrastar algunas informaciones obtenidas durante el proceso de observación, la realización del cuestionario ad hoc, así como ampliar algunas cuestiones que afloraban durante la investigación. Dicho instrumento se utilizó después de finalizar la intervención.

- Entrevista semiestructurada individual: se realizó, al finalizar la acción socioeducativa, al coordinador del proyecto. Dichas entrevistas se confeccionaron como un guion abierto, validado según el procedimiento de juicio de expertos, con el propósito de enfocar las cuestiones relevantes para el propósito del estudio.

- Análisis de documentos: se centró en el programa de la asignatura, el proyecto formativo, los 8 proyectos de ApS grupales diseñados por el alumnado universitario y de secundaria y los 25 informes reflexivos elaborados por el estudiantado universitario. Dicha estrategia se desarrolló durante las distintas etapas del proyecto: al inicio, durante el proceso y después de la implementación del 
proyecto.

Para el análisis de los datos cualitativos hemos partido de la técnica de codificación (Simons, 2011). Esta consiste en la reunión y análisis de los datos que referentes a temas, ideas, conceptos, interpretaciones y proposiciones con la intención de hallar contrastes y relaciones para generar conocimientos, interpretaciones y comprensiones de nuestro caso. De dicho proceso, realizado de forma manual, emergieron las distintas categorías (Tabla 3). Cada una de las categorías, aunque se presentan separadas para favorecer una descripción detallada de las mismas, están imbricadas en el proyecto objeto de indagación. En este estudio nos vamos a centrar en algunas de las categorías y subcategorías vinculadas a la percepción del estudiantado y del coordinador del proyecto sobre distintas dimensiones de la acción socioeducativa implementada.

\section{Tabla 3}

Categorías y subcategorías que emergieron durante el análisis de la información

\begin{tabular}{llll}
\hline Categorías & Subcategorías & & \\
\hline Conectar los contenidos & -Transferencia de conocimientos a contextos \\
teóricos de la asignatura a & reales. & & \\
situaciones de la vida real & -Proceso reflexivo. & \\
\hline Aprendizajes alcanzados & -Conocimiento de las organizaciones y \\
durante la implementación del & profesionales que participan en el proyecto. \\
proyecto de Aprendizaje- & -Planificación de acciones socioeducativas \\
Servicio & -Contenidos relacionados con la metodología ApS. \\
& -Adquisición de herramientas para su futuro \\
& profesional. \\
& -Dificultades y potencialidades que conlleva el \\
& trabajo en red. \\
\hline Desarrollo del compromiso & -Lectura crítica de la realidad. \\
social & -Interés por mejorar aspectos de la realidad. \\
& -Trabajar con jóvenes en contextos socialmente \\
& desfavorecidos \\
\hline
\end{tabular}

Fuente: elaboración propia. 
Con el propósito de contribuir a la veracidad de los resultados del estudio, se han llevado a cabo diferentes técnicas de triangulación (Taylor, S. J. \& Bogdan, 1992; Wood, P. \& Smith, 2018): a) Metodológica, para contrastar la información recogida a través de las diferentes técnicas e recogida de datos; b) Revisión y contraste de los datos proporcionados por las distintas fuentes de información para comprobar discrepancias y concordancias existentes; c) Grabación en vídeo de las observaciones y las entrevistas realizadas para, posteriormente, realizar la transcripción literal de todo lo expresado y acontecido, presentándose la misma a los informantes para obtener su validación, con el objetivo de eliminar el sesgo del investigador e intentar asegurar la credibilidad y replicabilidad de los datos (Álvarez, C. \& San Fabián, J 2012).

\section{Resultados}

\section{La Transferencia de Conocimientos a Situaciones de la Vida Real}

Los cambios vertiginosos que se están produciendo en los distintos ámbitos de la vida social, personal y profesional de los individuos en las sociedades actuales ha provocado que los contextos y escenarios sociales que rodean la vida de las nuevas generaciones en nada se parezcan a los escenarios y contextos que rodeaban el crecimiento de las generaciones del siglo XIX.

En la actualidad se exige a las instituciones educativas del nivel superior formar personas competentes, lo que hace que la formación deba apuntar al vínculo con el campo del desempeño profesional y la participación social, pues la sola actividad académica y la acumulación de conocimientos o de información acentúa las dificultades del estudiantado para insertarse en el ámbito laboral, social y político (Juliá, 2015). Desde esta perspectiva, el proyecto de ApS implementado muestra la pertinencia para poner en acción el enfoque de aprendizaje basado en competencias, ya que el proyecto favorecía la transferencia de algunos de los conceptos teóricos tratados en la asignatura: características de las organizaciones, las organizaciones que aprenden, liderazgo democrático en las organizaciones, instrumentos de planificación, etc. También posibilitaba el conocimiento de profesionales que desarrollan su trabajo en distintos ámbitos de la Educación Social, así como la adquisición de habilidades, actitudes y valores para diseñar y desarrollar estrategias y dinámicas para la intervención socioeducativa en un 
contexto real. Asuntos que conectaban con los intereses del estudiantado: "Nuestra profesión se desarrolla en múltiples ámbitos y durante nuestra formación sólo conocemos algunos de ellos de forma teórica" (Entrevista alumnado universitario 15), "He decidido participar en este proyecto porque me ofrece la posibilidad de conocer organizaciones y profesionales que trabajan en contextos reales" (Entrevista alumnado universitario 3), "Tenía ganas, después de tres años de teoría, de enfrentarme a un proyecto real para ver mis posibilidades en acción" (Entrevista alumnado universitario 11). Como se puede ver también en la siguiente cita:

En los cuatro años que llevamos de carrera, casi nunca, hemos llevado a cabo actividades donde se pudieran apreciar la teoría expuesta en clase, y creo que de la forma en que hemos trabajado en esta asignatura genera más aprendizaje que una clase magistral (Entrevista alumnado universitario 9).

De las respuestas emitidas por el estudiantado se desprende el interés por involucrarse en experiencias formativas que le ayude a encontrar sentido a las nociones teóricas tratadas en el ámbito académico, así como conocer organizaciones socioeducativas y profesionales que trabajen en la práctica y descubrir posibilidades para su futura inserción profesional. En este orden de ideas, el coordinador del proyecto planteaba:

La participación del estudiando universitario en el proyecto le ha posibilitado un conocimiento más profundo y real de organizaciones públicas y privadas insertas en el barrio. Organizaciones en las que ellos y ellas podrían trabajar en un futuro próximo. Al principio no eran conscientes de la complejidad implícita en la vida de las organizaciones sociales $\mathrm{y}$ de las distintas dificultades y potencialidades presentes en el desempeño de la práctica profesional. Desde esta perspectiva, su enganche en esta acción le ha servido para contrastar los conocimientos sobre las organizaciones adquiridos en la universidad con la realidad a la que se enfrentan dichas organizaciones en este contexto (Entrevista Coordinador proyecto). 
La última cuestión planteada por el coordinador del proyecto, conexión de los conocimientos académicos con la realidad vivenciada, es corroborada por el alumnado en el cuestionario ad hoc realizado (Figura 1), ya que cuando se le pide que valoren el grado de conexión entre los contenidos teóricos de la asignatura y el servicio a la comunidad, 23 de ellos/as, es decir el 92\%, responden que muy alto.

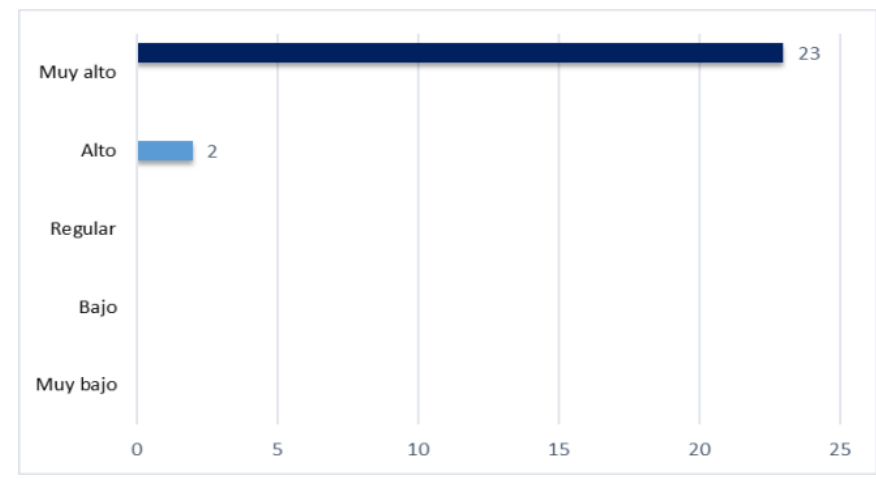

Figura 1. Valoración del grado de conexión entre los contenidos teóricos de la asignatura y el servicio a la comunidad. Fuente: elaboración propia.

También nos remite a los planteamientos sobre cómo se produce la transferencia de conocimientos (Jonnaert, P., Barrette, J., Masciotra, D. \& Yaya, 2008). Para dichos autores consiste en un proceso interactivo configurado por tres fases: contextualización-descontextualizaciónrecontextualización. Dado el carácter situacional y de problema abierto, el sujeto construye necesariamente una representación de la situación, que es transversal a todo el proceso de elaboración de la competencia. La competencia, desde una visión sociocultural, está conformada por las siguientes características: a) Una competencia se construye, no se transmite; b) Está situada en contextos y situaciones pertinentes con relación a las prácticas sociales, c) Establecidas, no puede plantearse descontextualizada; d) Requiere una práctica reflexiva; y e) Es temporalmente viable, no está definida de una vez por todas. 


\section{Aprendizajes Alcanzados Durante la Implementación del Proyecto de Aprendizaje-Servicio}

Los proyectos de ApS, como se ha expuesto anteriormente, se configuran como escenarios formativos expandidos que permiten generar un circulo virtuoso (Furco, 2003), a través de procesos reflexivos, entre los contenidos curriculares y el servicio: los aprendizajes sistemáticos enriquecen la calidad de la actividad social, y el servicio a la comunidad impacta en la formación integral y estimula una ulterior producción de conocimientos.

Asimismo, ofrecen la posibilidad de potenciar el rol protagónico del alumnado universitario, ya que los sitúa en el rol de agentes con responsabilidad de la toma de decisiones sobre distintas cuestiones que afloran en el acontecer de la experiencia desarrollada. En esta línea, los y las discentes involucrados en esta acción educativa exponían:

La posibilidad de intervenir en un escenario real me interesó mucho. Además, se nos ofrecía la posibilidad de aprender a diseñar proyectos, implementarlo y evaluarlos, esto es muy importante para desarrollar nuestra profesionalidad (Entrevista alumnado universitario 18).

Otra cuestión significativa es el establecimiento de ligaduras con otras agencias y profesionales, ello le ha posibilitado un conocimiento de las potencialidades y dificultades a las que se enfrentan en su quehacer cotidiano, así como la adquisición de múltiples técnicas y dinámicas para su futuro desarrollo profesional:

No sabía lo complicado que es poner en marcha un proyecto, todo lo que debes tener en cuenta para su diseño e implementación, las negociaciones que se tienen que mantener con los responsables de la organización, las limitaciones relacionadas con los presupuestos económicos e intereses políticos, etc. (Entrevista alumnado universitario 16)

Participar en este proyecto, donde intervenían muchos profesionales de distintos ámbitos, ha sido una experiencia muy rica de la que he aprendido muchas técnicas y dinámicas que no conocía. Muchas de ellas me servirán cuando trabaje como Educadora Social (Entrevista alumnado universitario 22). 


\section{Mayor-Aprendizaje Servicio y Educación Expandida}

En este escenario de educación expandida se producen múltiples aprendizajes, ya que está articulado a partir de distintos aportes profesionales, situaciones problemáticas sociales y personales a las que el alumnado tiene que dar respuesta, procesos de toma de decisiones vinculadas a la planificación de la acción: objetivos a conseguir, actividades a realizar, estrategias y técnicas a utilizar, etc. En este orden de ideas, cuando se le pregunta al alumnado por los aprendizajes alcanzados (Figura 2) exponen lo siguiente: a) El 76\% (19 de 25) valoran como muy alto los aprendizajes conectados al conocimiento de las dificultades y potencialidades de trabajo colaborativo entre distintas organizaciones y profesionales; b) El 92\% /23 de 25) aprecian como muy alto sus aprendizajes sobre la metodología ApS; c) El 88\% (22 de 25) estiman como muy alto los conocimientos adquiridos sobre técnicas y dinámicas para trabajar con grupos; d) El 92\% (23 de 25) valoran como muy alto los conocimientos alcanzados vinculados a los aspectos teóricos del ApS; y e) El 80\% (20 de 25) asignan un valor muy alto a los conocimientos logrados sobre las organizaciones que implementan proyectos socioeducativos.

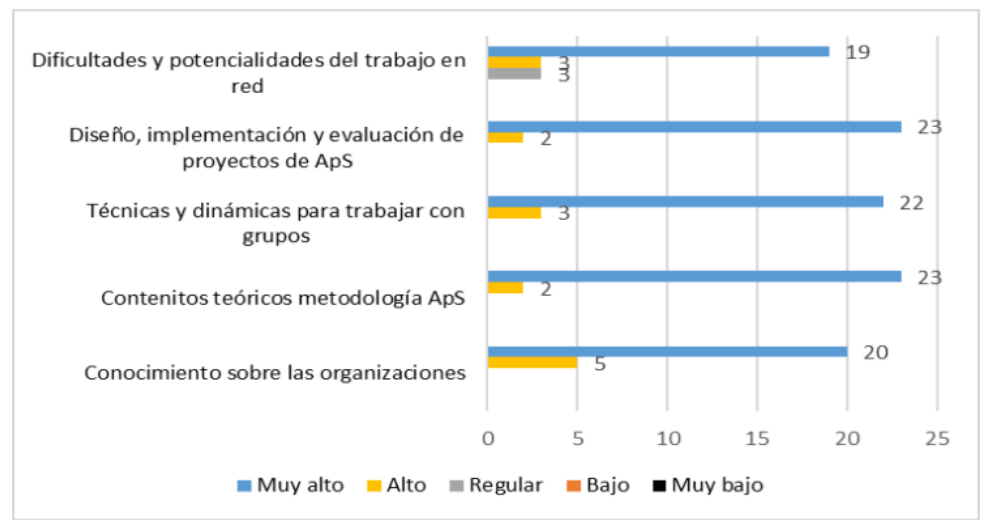

Figura 2. Valoración de los aprendizajes alcanzados. Fuente: elaboración propia. 
Los valores altos, utilizados por el estudiantado para explicitar los tipos de aprendizajes alcanzados que consideran significativos, son congruentes con los valores atribuidos a su nivel de satisfacción alcanzado (Figura 3) en esta acción socioeducativa:

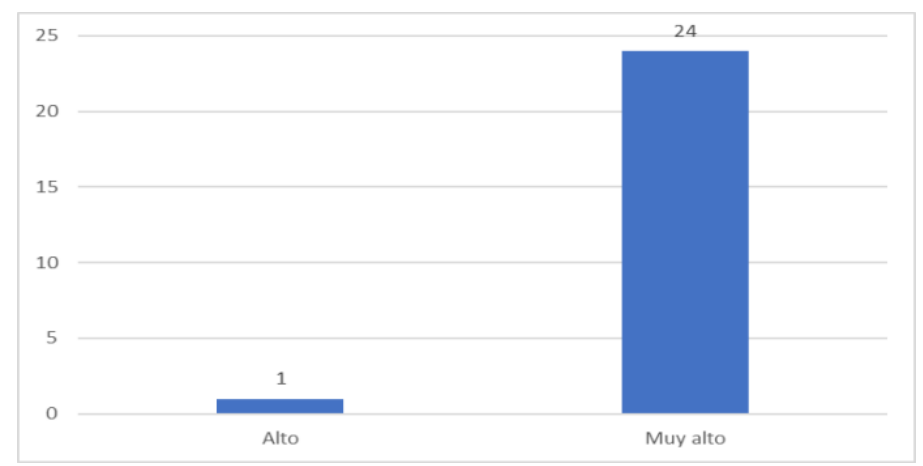

Figura 3. Nivel de satisfacción alcanzado por el alumnado implicado en el proyecto. Fuente: elaboración propia.

\section{Aprendizaje-Servicio y Desarrollo del Compromiso Social}

En la Comunicación de la Comisión Europea sobre una agenda renovada de la UE para la educación superior se expone:

(...) Para contrarrestar la creciente polarización de nuestra sociedad y la desconfianza hacia las instituciones democráticas, cada uno de nosotros, incluidos el personal y los estudiantes de educación superior, debemos comprometernos más activamente con las comunidades que nos rodean y promover la inclusión social y la movilidad (Comisión Europea, p. 2).

Para promover el compromiso social en el estudiantado universitario hay que generar situaciones de aprendizaje donde los contenidos conceptuales se materialicen en comportamientos y actitudes. Como plantean Fuentes y Pozo (Fuentes, J. L. \& Pozo, , p. 120): "El educador no puede limitarse a presentar valores y a discutir sobre ellos, sino que debe promover actividades que permitan poner en práctica tales valores, incorporándolos al repertorio comportamental habitual de los estudiantes". En este sentido, diferentes 


\section{Mayor-Aprendizaje Servicio y Educación Expandida}

estudios han mostrado que el $\mathrm{ApS}$ es una propuesta metodológica especialmente efectiva para poner en práctica contenidos teóricos a la vez que se promueve la solidaridad, la empatía, el compromiso social, etc. (Folgueiras, P. \& Martínez, 2009).

En línea con lo anterior, durante el desarrollo del proyecto del estudiantado universitario pudo conocer de primera mano algunos asuntos de los que no eran conscientes.

Durante la implementación del proyecto los y las discentes estuvieron conociendo a representantes de entidades sociales que trabajan en el barrio. En el transcurso de los itinerarios realizados, acompañados por jóvenes de enseñanza secundaria que habitaban en el lugar, pudieron observar situaciones de pobreza que vivían algunas familias, el abandono institucional en cuanto a la limpieza de los espacios y alumbrado público, el deterioro de algunos edificios y del patío del centro de educación primaria, los problemas de convivencia entre familias pertenecientes a distintas culturas (gitanos y árabes), los proyectos socioeducativos implementados por las entidades sociales, etc. Posteriormente estuvieron analizando y debatiendo sobre la experiencia vivida (Diario de observación).

Este ejercicio cognitivo, afectivo, ético y relacional favoreció la problematización y toma de conciencia de la realidad donde se decidió intervenir, y la justificación de la acción socioeducativa a desarrollar. Una actuación de carácter educativo y político que sirvió para situar al estudiantado como agentes con potencial de cambio individual y social, favoreciendo el desarrollo y puesta en acción de su compromiso social. Así lo manifestaba el coordinador del proyecto:

El ApS, como práctica experiencial y reflexiva, favorece la formación en valores conectados a la justicia social, responsabilidad social, la solidaridad, etc., puesto que, para su puesta en acción, necesita de un análisis crítico de las necesidades a las que pretende dar una respuesta. Los descubrimientos alcanzados durante el proceso ayudan, por un lado, a enriquecer los conocimientos previos del alumnado, favoreciendo la toma de conciencia crítica de la realidad analizada y, por otro lado, al tener que diseñar acciones orientadas a mejorar esa realidad, se posicionan como agentes de cambio. Ello comporta un 
desarrollo de su compromiso social (Entrevista Coordinador proyecto).

\section{Conclusiones}

El objetivo que orienta este trabajo es conocer la percepción que tiene el estudiantado universitario y el coordinador del proyecto de ApS. Teniendo en consideración los resultados alcanzados, se plantean varias conclusiones.

El ApS, como práctica educativa, se puede situar en línea con el enfoque de enseñanza-aprendizaje basado en adquisición y desarrollo de competencias (Pérez, 2012) o pensamiento práctico (Schön, 1992), entendido como un proceso donde los y las aprendices ponen en acción conocimientos, habilidades, actitudes y valores para resolver situaciones problemáticas en contextos reales. Dicho enfoque supone un salto cualitativo en la concepción de la conexión teoría-práctica, ya que la visión escolástica plantea una relación lineal y unidireccional, donde la práctica está subordinada a la teoría. Afirmaciones que han sido refutadas por distintas investigaciones implementadas en lo últimos años, así como la experiencia desarrollada por distintos profesionales, donde se pone de manifiesto que el mero aprendizaje académico de contenidos teóricos no garantiza la formación y permanencia de las competencias para la vida (Pérez, 2012).

En línea con lo anterior, y teniendo en cuenta los hallazgos alcanzados, podemos plantear que el ApS se configura como un escenario formativo que favorece la retroalimentación de los contenidos teóricos con las actividades derivadas del servicio a la comunidad. En este sentido, podemos plantear que las prácticas de ApS permiten al estudiantado vincular el pensamiento y la acción, así como la reconstrucción de lo aprendido mediante procesos reflexivos sistemáticos que sirven para orientar y conceptualizar el acontecer de la experiencia (Páez, M. \& Puig, 2013).

La reflexión sistemática, antes, durante y después del proceso vivido, le sirve a los y las discentes para tomar distancia de lo acontecido, requisito necesario para tomar conciencia de los aprendizajes alcanzados en relación con las actividades del servicio, los contenidos curriculares y con los sentimientos despertados. 


\section{Mayor-Aprendizaje Servicio y Educación Expandida}

En general, los aprendizajes alcanzados son de naturaleza experiencial. El aprendizaje experiencial (Kolb,1984) se basa en una idea holística del aprendizaje, en tanto que aborda el desarrollo global de la persona enfatizando el protagonismo del aprendiz en la creación de sentido y significado en su relación con el mundo (Díaz, 2015).

Tomando en consideración lo expresado anteriormente, los aprendizajes obtenidos durante el proyecto de ApS implementado se pueden encuadrar en el modelo de aprendizaje propuesto por Kolb (Kolb), ya que el alumnado a partir de su experiencia, y teniendo en cuenta los contenidos de la asignatura, pudo observar de forma reflexiva el funcionamiento de distintas organizaciones, los proyectos que implementaban los profesionales pertenecientes a las mismas, las posibilidades y límites encontrados, analizar la realidad sociocultural donde se iba a poner en acción sus actuaciones, implementar proyectos junto a otros agentes socioeducativos, etc.; conocimientos que sirvieron para poder establecer un diálogo reflexivo entre la teoría y la práctica, reconstruyendo así algunas concepciones previas y pudiendo dotar de sentido a los aprendizajes alcanzados.

Para el estudiantado universitario implicado en prácticas educativas con enfoque ApS supone el contacto con un proceso formativo configurado como un escenario que favorece la adquisición de múltiples aprendizajes: estructuras de las distintas organizaciones, historia, funciones, relaciones establecidas, proyectos implementados, influencia en la población, etc. Aprendizajes propiciados por su relación con profesionales de su ámbito profesional, así como por su enganche en la articulación de acciones socioeducativas que conllevan la puesta en acción de sus saberes alcanzados y dar respuesta a los interrogantes que van emergiendo en el proceso. Dichos asuntos sirvieron para mantener el interés del alumnado a lo largo del proceso.

Otra cuestión significativa son los aprendizajes útiles y relevantes sobre organizaciones reales, no contemplados en el programa de la asignatura, obtenidos a partir de abrir espacios de educación expandida donde participan profesionales pertenecientes a distintas instituciones públicas y entidades sociales.

En cuanto al desarrollo del compromiso social, los proyectos de ApS se articulan a partir de una mirada crítica a las necesidades sociales 
detectadas para realizar una contribución positiva a la realidad. Ello implica situar al estudiantado como observadores reflexivos y críticos con la intencionalidad de acercarse a las causas que sostienen las carencias identificadas (Manzano, 2011). Posteriormente, los y las discentes, asumiendo un rol protagónico junto a otros agentes socioeducativos, diseñan mundos posibles que den respuestas a las necesidades detectadas. Un proceso metacognitivo, afectivo y relacional, donde se pone en acción el compromiso social del alumnado a través de su implicación en la construcción de un proyecto socioeducativo y político orientado a mejorar aspectos de la realidad social.

En el transcurso de dicha acción se ha posibilitado el tránsito de una conciencia mágica, donde la aprehensión de la realidad es superficial; a una conciencia crítica como resultado de la captación profunda de los nexos causales (Freire, 2002).

Como conclusión, en este estudio se ha evidenciado que el ApS promueve la creación de escenarios de educación expandida donde participan agencias y agentes pertenecientes al ámbito de la educación formal y no formal. Escenarios construidos a partir de la intersección de saberes académicos y experienciales y la articulación de acciones socioeducativas en contextos reales orientadas a mejorar aspectos de la realidad. Todo ello favorece la conexión teoría-práctica, la adquisición de aprendizajes múltiples y multidireccionales y el desarrollo del compromiso social de los y las participantes.

En cuanto con los límites de esta investigación, cabe señalar que una limitación es su carácter de estudio de caso a partir de un fenómeno singular, aunque los resultados pueden significar una nueva oportunidad para modificar antiguas generalizaciones (Stake, 2005), así como la transferencia de los resultados a otros contextos, dependiendo del grado de similitud entre ambos (Guba, 1989).

Respecto a posibles líneas de investigación, se propone avanzar en los siguientes asuntos:

- Analizar las razones que sostienen la implicación de los distintos agentes participantes en las prácticas de ApS.

- Explorar la influencia de los proyectos de ApS en la adquisición y desarrollo de las competencias del alumnado universitario. 
70 Mayor - Aprendizaje Servicio y Educación Expandida

\section{Referencias}

Álvarez, C. \& San Fabián, J. L. (2012). La elección del estudio de caso en investigación educativa. Gazeta de Antropología, 28(1), 1-12. Recuperado de http://www.ugr.es/ pwlac/G28_14Carmen_AlvarezJoseLuis_SanFabian.html

Asociación Internacional de Ciudades Educadoras (2014). Ciudad, inclusión social y educación. Barcelona: Ayuntamiento de Barcelona.

Carbonell, J. (2016). Pedagogías del S. XXI. Alternativas para la innovación educativa. Barcelona: Octaedro.

Caride, J. A. (2006). La educación social en la acción comunitaria. En Úcar, X. y Llena, A. (coords.) Miradas y diálogos en torno a la acción comunitaria (pp. 157-190). Barcelona: Graó.

Chiva-Bartoll, O. \& Gíl-Gómez, J. (2018). (eds.). Aprendizaje-servicio universitario. Modelos de intervención e investigación en la formación inicial del docente. Barcelona: Octaedro.

Coll, C. (2013). El currículo escolar en el marco de la nueva ecología del aprendizaje. Aula de Innovación Educativa, 219, 31-36.

Comisión Europea (2017). Sobre una agenda renovada de la Unión Europea para la educación superior. COM (2017) 247 final. Recuperado de https://eurlex.europa.eu/legal-content/ES/TXT/PDF/?uri=CELEX:52017DC0247

Contreras, A., Berríos, V., Herrada, M., Robles, M. C. \& Rubio, X. (2012). Resumen de estudio. Resultados del Aprendizaje Servicio en la U C desde la mirada de sus actores: docentes, estudiantes y socios comunitarios (2005-2011).

Chile: Pontificia Universidad Católica. Recuperado de http://desarrollodocente.uc.cl/Investigacion-general/investigacion.html

Dewey, J. (1950). Las escuelas de mañana. Buenos Aires: Losada.

Díaz, F. (2015). Estrategias para el desarrollo de competencias en Educación Superior. I Encuentro Internacional Universitario. El currículo por competencias en la Educación Superior, 63-86- Extraído el 21 de enero de 2019 desde http://cdn02.pucp.education/academico/2015/09/01094909/curriculo_comp etencias.pdf

Folgueiras, P. \& Martínez, M. (2009). El desarrollo de competencias en la universidad a través del Aprendizaje y Servicio Solidario. Revista 
Interamericana de Educación para la Democracia, 2(1), 55-76.

Recuperado de http://diposit.ub.edu/dspace/handle/2445/21972

Folgueiras, P., Luna, E. \& Escofet, G. (2013). Aprendizaje y servicio: estudio del grado de satisfacción de estudiantes universitarios. Revista de Educación, 362, 159-185. doi: 10.4438/1988-592X-RE-2011-362-157

Freire, P. (2002). La educación como práctica de la libertad. España: Siglo XXI.

Freire. P. (1994). Educación y participación comunitaria. En M. Castells (coord.). Nuevas perspectivas críticas de la educación (pp. 83-96). Barcelona: Paidós.

Fuentes, J. L. \& Pozo, A. (2019). Promoción de la competencia ética y el compromiso cívico y su evaluación en los proyectos de AprendizajeServicio. En Ruiz-Corbella y J. García-Gutiérrez. Aprendizaje-Servicio. Los retos de la evaluación, (pp. 117-134). Madrid: Narcea.

Furco, A. (2003). El impacto educacional del aprendizaje-servicio. ¿Qué sabemos a partir de la investigación? University of California-Berkeley. Recuperado de http://roserbatlle.files.wordpress.com/2009/02/resultats-investigacioaps-furco-modo-de-compatibilidad.pdf

Furco, A. (2007). Impactos de los proyectos de aprendizaje-servicio. En A. González (coord.). Antología 1997-2007. Seminarios Internacionales de Aprendizaje y Servicio Solidario (pp. 175-183). Buenos Aires: EUDEBA. Editorial Universitaria de Buenos Aires. Recuperado de http://repositorio.educacion.gov.ar:8080/dspace/bitstream/handle/12345678 9/94942/2007_antologia_10.pdf?sequence=1

García, D. \& Lalueza, J. L. (2019). Procesos de aprendizaje e identidad en aprendizaje-servicio universitario: una revisión teórica. Educación XX1, 22(2), 45-68, doi: 10.5944/educXX1.22716

García, T., Sánchez, M. S. \& Mayor, D. (2012). La vinculación teoría-práctica en un espacio inclusivo: escuela-comunidad-universidad. Revista de educación inclusiva, 5(1), 107-120.

Gimeno, J. (2005). La educación obligatoria: su sentido educativo y social ( $3^{\mathrm{a}} \mathrm{ed}$.). Madrid: Morata.

Guba, E. (1989). Criterios de credibilidad en la investigación naturalista. En J. Gimeno y A. I. Pérez. (coords.). La enseñanza: su teoría y su práctica (pp. 148-165). Madrid: Akal.

Jonnaert, P., Barrette, J., Masciotra, D. \& Yaya, M. (2008). La competencia como organizadora de los programas de formación: hacia un desempeño 
competente. Profesorado. Revista de curriculum y formación del profesorado, 12(3), 1-32. Recuperado de https://www.ugr.es/ recfpro/rev123ART3.pdf

Jouannet, C., Ponce, C. \& Contreras, A. (2012). Impacto de la metodología de Aprendizaje-Servicio según la percepción de los docentes. En M. A. Herrero y M. N. Tapia (comps.). Actas de las II Jornadas de Investigadores sobre Aprendizaje-Servicio. Buenos Aires: CLAYSS. Recuperado de http://www.clayss.org/06_investigacion/jornadas/Libro_II-JornadaInvestigadores-Aprendizaje-Servicio/CII-02_Jouannet-\%20PonceContreras.pdf

Juliá, M. T. (2015). Competencias generales de la formación universitaria: Aportes desde la equidad. I Encuentro Internacional Universitario. El currículo por competencias en la Educación Superior (pp. 39-62). Perú: Pontificia Universidad Católica de Perú.

Kolb, D. A. (1984). Experiential learning: experience as the source of learning and development. Englewoods Cliffs: Prentice Hall.

Manzano, V. (2011). La Universidad Comprometida. Barcelona: Hipatia.

Martínez, J. B. \& Fernández, E. (2018). Ecologías del aprendizaje. Educación expandida en contextos múltiples. Madrid: Morata.

Máximo, E. (2010). Aprendizaje-Servicio en América Latina: apuntes sobre pasado y presente. Revista Científica Tzhoecoen, 3(5), 108-125. Recuperado de http://www.clayss.org.ar/archivos/TZHOECOEN-5.pdf

Mayor, D. (2018). Prácticas de Aprendizaje-Servicio como escenarios de confluencia entre la educación escolar y social. Revista Iberoamericana de Educación, 76, 35-56. Extraído el 5 de marzo de 2019 desde https://rieoei.org/RIE/article/view/2847

Mayor, D. \& Rodríguez, D. (2016). Aprendizaje-Servicio: construyendo espacios de intersección entre la escuela-comunidad-universidad. Profesorado. Revista de currículum y formación del profesorado, 19(1), 262-279. Recuperado de http://www.ugr.es/ recfpro/rev191ART11.pdf

Mayor, D. \& Rodríguez, D. (2017). Aprendizaje-Servicio y práctica docente: una relación para el cambio educativo. Revista de Investigación Educativa, 32(2), 535-552. DOI: http://dx.doi.org/10.6018/rie.34.2.231401

Mella, I. Santos, M. A. \& Malherio, J. M. (2015). Aprendizaje-Servicio y rendimiento académico del alumnado universitario. Revista de Estudios e 
Investigación en Psicología y Educación, 1(12), 1-5. doi:

10.17979/reipe.2015.0.12.569

Montes, R., Tapia, M., \& Yaber, L. (2011). Manual para docentes y estudiantes solidarios. Buenos Aires: CLAYSS. Recuperado de http://www.clayss.org.ar/04_publicaciones/Natura2013.pdf

Muñoz, J. L., Rodríguez, D. \& Barrera, A. (2013). Herramientas para la mejora de las organizaciones educativas y su relación con el entorno. Perspectiva Educacional, 52(1), 97-123.

Olsen, E. (1951). La escuela y la comunidad. México: Unión Tipográfica Editorial Hispano-Americana.

Páez, M. \& Puig, J. M. (2013). La reflexión en el aprendizaje-servicio. Revista Internacional de Educación para la Justicia Social, 2(2), 13-32.

Recuperado de https://revistas.uam.es/riejs/article/view/370

Pérez, A. I. (2012). Educarse en la era digital. Madrid. Morata.

Puig, J. M. \& Palos, J. (2006). Rasgos pedagógicos del aprendizaje-servicio. Cuadernos de Pedagogía, 357, 60-63.

Puig, J. M., Batlle, R., Bosch, C. \& Palos, J. (2007). Aprendizaje servicio. Educar para la ciudadanía. Madrid: Octaedro.

Rodríguez, M. \& Ordoñez, R. (2015). Una experiencia de Aprendizaje-Servicio en comunidades de aprendizaje. Profesorado. Revista de Currículum y Formación del Profesorado, 19(1), 314-333. Recuperado de https://recyt.fecyt.es/index.php/profesorado/article/view/41039

Ruiz-Corbella, M. \& García-Gutiérrez, J. (2019). Aprendizaje-Servicio. Los retos de la evaluación. Madrid: Narcea.

Schön, D. (1992). La formación de profesionales reflexivos. Hacia un nuevo diseño de la formación y el aprendizaje de las profesiones. Barcelona: Paidós.

Simons, H. (2011). El estudio de caso: Teoría y práctica. Madrid: Morata.

Stake, R.E. (2010). Investigación con estudio de casos. Madrid: Morata.

Subirats, J. (2002). El factor proximidad y de comunidad en las políticas educativas. En J. Subirats (coord.). Gobierno Local y Educación. La importancia del territorio y la comunidad en el papel de la escuela (pp. 21-50). Barcelona: Ariel.

Taylor, S. J. \& Bogdan, R. (1992). Introducción a los métodos cualitativos de investigación. Barcelona: Paidós. 
Trilla, J. (2004). La educación no formal y la ciudad educadora. En H. Casanova y C. Lozano. Educación, universidad y sociedad: el vínculo crítico. Barcelona: Universidad de Barcelona.

Trilla, J. (2005). La idea de ciudad educadora y escuela. IDEP-Revista Educación y Ciudad, 7, 75-106. Recuperado de http://idep.edu.co/pdf/revista/Revista07.pdf

UNESCO. (1996). La educación encierra un tesoro. Madrid: Santillana.

Universidad de Almería. (2018). Programa de la asignatura de Dirección y Gestión Pedagógica de Organizaciones Socioeducativas. Recuperado de http://cvirtual.ual.es/guiado/servlet/bin?id=25439_CAS

Wood, P. \& Smith, J. (2018). Investigar en Educación. Conceptos básicos y metodología para desarrollar proyectos de investigación. Madrid: Narcea.

Zeichner, K. (2010). Nuevas epistemologías en formación del profesorado.

Repensando las conexiones entre las asignaturas del campus y las experiencias de prácticas en la formación del profesorado en la universidad. Revista Interuniversitaria de Formación del Profesorado, 68(24,2), 123 149. Recuperado de http://maxconn.aufop.com/aufop/uploaded_files/articulos/1279237815.pdf ZEMOS. (2012). Educación expandida. Creative Commons. Recuperado de http://www.zemos98.org/descargas/educacion_expandida-ZEMOS98.pdf

Domingo Mayor Paredes es profesor del Departamento de Educación de la Universidad de Almería

Contact Address: Departamento de Educación, Universidad de Almería, España.

Email: domingomayor1960@gmail.com 